\title{
Long-Term Effects of Altered Photoperiod During Pregnancy on Liver Gene Expression of the Progeny
}

\author{
Pamela Carmona',2, Bárbara Pérez', Carlos Trujillo1,3, Gabriel Espinosa', \\ Fernando Miranda', Natalia Mendez ${ }^{4}$, Claudia Torres-Farfan', Hans G. Richter ${ }^{4}$, \\ Karina Vergara ${ }^{4}$, Priscilla Brebi ${ }^{2,5}$ and José Sarmiento ${ }^{1 *}$
}

' Laboratorio de Cronoinmunología, Instituto de Fisiología, Facultad de Medicina, Universidad Austral de Chile, Valdivia, Chile, ${ }^{2}$ Programa de Doctorado en Ciencias Médicas, Universidad de La Frontera, Temuco, Chile, ${ }^{3}$ Programa de Doctorado en Ciencias Veterinarias, Universidad Austral de Chile, Valdivia, Chile, ${ }^{4}$ Laboratorio de Cronobiología del Desarrollo, Instituto de Anatomía, Histología y Patología, Facultad de Medicina, Universidad Austral de Chile, Valdivia, Chile, ${ }^{5}$ Laboratorio de Patología Molecular, Departamento de Patología, Facultad de Medicina, Universidad de La Frontera, Temuco, Chile

Experimental and epidemiological studies have revealed a relationship between an adverse intrauterine environment and chronic non-communicable disease (NCD) like cardiovascular disease (CVD) in adulthood. An important risk factor for CVD is the deregulation of the fibrinolytic system particularly high levels of expression of plasminogen activator inhibitor 1 (Pai-1). Chronic exposure to altered photoperiod disrupts the circadian organization of physiology in the pregnant female, known as gestational chronodisruption, and cause long-term effects on the adult offspring's circadian physiology. The Pai-1 expression is regulated by the molecular components of the circadian system, termed clock genes. The present study aimed to evaluate the long-term effects of chronic photoperiod shifts (CPS) during pregnancy on the expression of the clock genes and the fibrinolytic system in the liver of adult male offspring. Our results using an animal model demonstrated statistically significant differences at the transcriptional level in males gestated under CPS. At 90 days of postnatal age, the liver transcript levels of the clock gene Bmal1 were downregulated, whereas Rora, Ror $\gamma, \mathrm{Nfil}$, and Pai-1 were upregulated. Our data indicate that CPS during pregnancy affects gene expression in the liver of male adult progeny, showing that alteration of the photoperiod in the mother's environment leads to persistent effects in the offspring. In conclusion, these results reveal for the first time the long-term effects of gestational chronodisruption on the transcriptional activity of one well-established risk factor associated with CVD in the adult male offspring.

Keywords: cardiovascular disease, fibrinolytic system, Pai-1, clock genes, gestational chronodisruption, DOHaD

Abbreviations: CCG, clock-controlled gene; CPS, chronic photoperiod shifts; CVD, cardiovascular disease; E18, embryonic day 18; LD, light dark; NCD, non-communicable disease; Pai-1, plasminogen activator inhibitor-1; Plg, plasminogen; P1, postnatal day 1; P60, postnatal day 60; P90, postnatal day 90; P120, postnatal day 120; ROR, retinoid-related orphan receptor; RORE, ROR-response elements; tPA, tissue Plasminogen Activator; uPA, urokinase Plasminogen Activator; ZT, zeitgeber time. 


\section{INTRODUCTION}

The environment during early life influences the risk of developing pathophysiological processes later; the field recognized as the developmental origins of health and disease (DOHaD) (Calkins et al., 2011; Capra et al., 2013; Hanson and Gluckman, 2014). In particular shows an association with NCDs (Fowden et al., 2006; Marsh et al., 2011) as CVD (Thompson and Trask, 2016).

In modern society, exposure to environmental light at night (e.g., chronic shift work, work at night), disarranging the internal biological clock; thus producing a significant disturbance of the circadian organization of physiology known as chronodisruption (Erren and Reiter, 2009). Circadian rhythms are intrinsic biological oscillations with a 24 -h period driven by the circadian timing system, coordinating physiology and behavior with the daily light/dark cycle (Mazzoccoli et al., 2012; Partch et al., 2014; Tarquini and Mazzoccoli, 2017). This system is organized by the central clock, located in the suprachiasmatic nucleus; which is entrained by the light/dark cycle as a dominant signal, in addition to several peripheral clocks located throughout the body. At the cell level, circadian rhythmicity relies on clock gene expression in central and accessory interlocking transcription/translation feedback loops (TTFL) (Mohawk et al., 2013; Curtis et al., 2014; Takahashi, 2016). In turn, these core clock genes promote the expression of downstream genes (CCGs) (Albrecht, 2012; Liu and Chu, 2013). Significant changes in the expression of clock genes can affect physiological processes controlled by the biological clock and have been associated with the development of NCDs (Plano et al., 2017; Touitou et al., 2017).

The misalignment of the maternal circadian system (gestational chronodisruption) impacts fetal health (SerónFerré et al., 2012, 2013). This field is of great interest because of the potential long-term effects on the adult offspring's health and disease status (Amaral et al., 2014; Varcoe et al., 2017; Richter et al., 2018). The available evidence has demonstrated different consequences of chronodisruption on maternal physiology (Gatford et al., 2019). In animal model, the maternal exposure to CPS disrupted the biological clocks in the pregnant female, altering physiological parameters throughout gestation such as the circadian profile of plasma hormones, changes in the liver metabolic gene expression and alterations in the clock gene expression profile (Varcoe et al., 2013; Mendez et al., 2016). Meanwhile, in the adult offspring gestational chronodisruption induced effects such as hyperleptinemia, hyperinsulinemia, impaired glucose tolerance (Varcoe et al., 2011); alterations in the plasma circadian profile of melatonin and corticosterone (Mendez et al., 2016); as well as alteration of adrenal endocrine messengers. In fact, there is strong evidence suggesting that the adrenal gland loses the ability to respond to ACTH (Mendez et al., 2016; Salazar et al., 2018). Given that the endocrine adrenal outputs play a key role in the development and entrainment of the fetal clock in the suprachiasmatic nucleus (Čečmanová et al., 2019), coordinating metabolic responses and acting as time-giving signals to other peripheral circadian oscillators such as the liver (Pezük et al., 2012); long-term alterations of adrenal function can lead to multiple pathophysiological processes.

The liver is a well-described peripheral clock and as such, its physiology is controlled by circadian rhythms, the clock regulates the transcription of CCGs that participate in a wide array of the physiological process in the liver (Reinke and Asher, 2016; Tahara and Shibata, 2016; Zwighaft et al., 2016) and the evidence supports that synchronized liver clockwork machinery develops gradually during ontogenesis (Sumová et al., 2008). On the other hand, the liver is the major site of Pai-1 synthesis, being regulated transcriptionally by endocrine signals of the adrenal gland, which in turn strongly responds to light input (Dimova and Kietzmann, 2008; Aoshima et al., 2014). Also, Pai-1 is a CCG (Haus, 2007) and its expression is upregulated through binding of the CLOCK: BMAL heterodimer to E-box sites of the Pai-1 gene promoter region (Maemura et al., 2000; Schoenhard et al., 2003; Ohkura et al., 2006). Also, the transcription of Pai1 is promoted by ROR $\alpha$ and repressed by REV-ERB $\alpha$ acting on RORE sites (Wang et al., 2006), all of them important members of the clock molecular machinery. Of note, epidemiological studies identify PAI-1 as a risk factor for CVD (Tofler et al., 2016; Jung et al., 2018).

Our hypothesis is that gestational chronodisruption promotes changes in the adult offspring, specifically, alterations of the regulation of molecular machinery of the liver clock genes; which in turn regulate the transcriptional pattern of the Pai-1 in the liver. To test our hypothesis, we used a rat model of gestational chronodisruption. Our specific aims were to investigate the impact of prenatal CPS in the liver of adult male progeny on (1) clock gene transcription patterns; and (2) the fibrinolytic system, particularly in the Pai-1 transcriptional levels.

\section{MATERIALS AND METHODS}

\section{Animals}

Animal handling and care followed the Guide for the Care and Use of Laboratory Animals of the Institute for Laboratory Animal Research of the National Research Council. The protocols were approved by the Bioethics Commission of the Universidad Austral de Chile (CBA number 267/2016).

The animals were maintained in a control (standard) photoperiod [12 h light, $12 \mathrm{~h}$ dark cycle; lights on at 7:00 AM (ZT0), lights off at 7:00 PM (ZT12)]; $\sim 400$ lux at the head level, temperature $\left(18-20^{\circ} \mathrm{C}\right)$, humidity $(\sim 48 \%)$, food and water were available ad libitum (Mendez et al., 2016; Salazar et al., 2018). Sprague Dawley rats (obtained from Charles River Laboratories International Inc.) were mated and raised in our animal facility. Timed-pregnant females were used in the study, and the day in which spermatozoa were observed in the smear of the vaginal contents was considered embryonic day 0 (E0). The pregnant females were separated by weight pairing and allocated to the following two photoperiods: light/dark (LD; control photoperiod) and CPS, using the same protocol reported by Mendez et al. (2016). Briefly, pregnant females were exposed to lighting 
schedule manipulation every 3-4 days, reversing the photoperiod completely, during 18 days of pregnancy (Figure 1). At 18 days of gestation, the mothers returned to a control 24-h photoperiod (12:12, lights on at ZT0) and continued in this photoperiod thereafter.

\section{Effects of Gestational Chronodisruption on Daily Rhythms and mRNA Expression in Adult Offspring}

After birth, both dams and pups from each pregnancy condition (LD; $n=12$ and CPS; $n=6$ mothers) were kept under control photoperiod and litters were weighed at postnatal age
1 day (P1) and homogenized to 10 individuals (five males and females), in order to avoid variations in weight gain. Pups were weaned at 21 days old, with the males being raised in the control photoperiod (LD) to be studied at P90 (LD and CPS, $n=30$ each group).

Body weight was measured from 30 days old, every 7 days. Males from each pregnancy condition were euthanized at P90 every $4 \mathrm{~h}$ for six samplings over $20 \mathrm{~h}$, in LD and CPS $(n=5 /$ each time point), starting at ZT1 and ending at ZT21. To avoid litter effects, each clock time point contained animals from different mothers; thus, no siblings were used at the same time point. Briefly, male rats were deeply anesthetized (isoflurane 3.5\%, Baxter Laboratories), a blood sample was collected from the

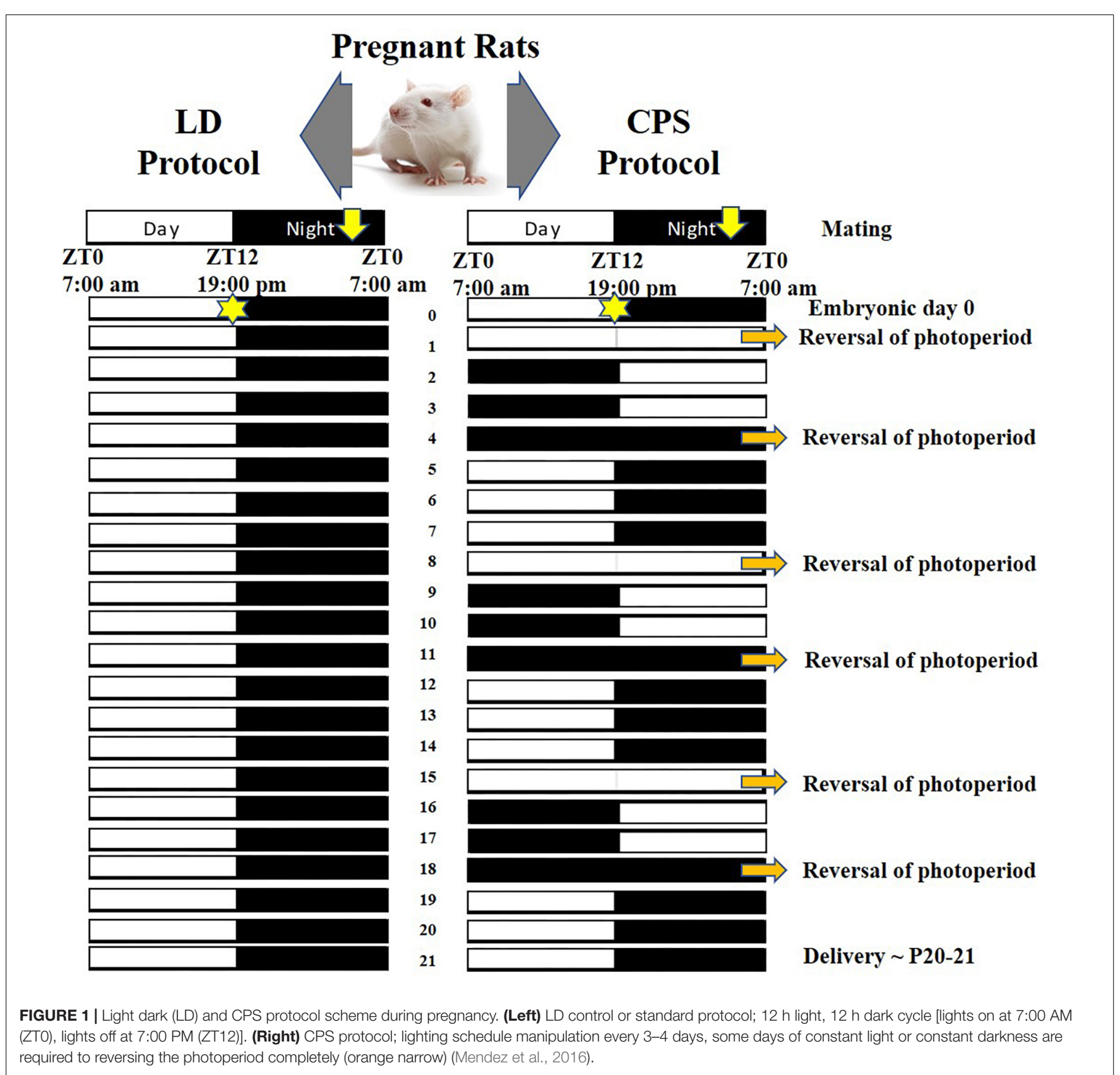


vena cava, and then an overdose of T61 $(0.5 \mathrm{ml} / \mathrm{kg}$ body weight; Merck Animal Health, Intervet Canada Corp., Kirkland, QC, Canada) was delivered at the same site. Organs were collected, weighed and stored in RNA stabilization solution (RNAlater ${ }^{\mathrm{TM}}$ Invitrogen) at $4^{\circ} \mathrm{C}$ for $24 \mathrm{~h}$ and subsequently at $-20^{\circ} \mathrm{C}$ in our tissue bank.

\section{RNA Extraction and Quantitative Real-Time PCR (RT-PCR) Analysis of the Liver}

Relative quantification by RT-PCR was used to evaluate the mRNA expression of clock genes and fibrinolytic system genes at P90. Total RNA was extracted using the SV Total RNA Isolation System (Promega) according to the manufacturer's instructions. The amount of $2.0 \mu \mathrm{g}$ of total RNA was reverse transcribed using random primers (Promega) and MLT-V reverse transcriptase (Promega). RT-PCR was performed using primers described in Supplementary Table 1 and KAPA SYBR FAST quantitative PCR master mix (Kapa Biosystems, Inc.). Quantitative PCR was carried out in a Rotor-Gene Q real-time platform (QIAGEN). Serial dilutions of cDNA were amplified by real-time PCR using specific primers for target and reference gene and determining template dilution for the sample's measurements. A melting curve analysis was performed on each sample after the final cycle to ensure that a single product was obtained. Relative amounts of all mRNAs were calculated by the comparative $\Delta \Delta$ cycle threshold method using the equation $2^{-\Delta \Delta \mathrm{Ct}}$ to linearize the data and then perform the statistical analysis (Livak and Schmittgen, 2001) and normalized to the corresponding 18S-rRNA housekeeping level. For the analysis of daily rhythms we follow the Guidelines for Genome-Scale Analysis of Biological Rhythms (Hughes et al., 2017) and three independent methods were used; single cosinor (Refinetti et al., 2007), JTK_Cycle (Hughes et al., 2010), and RAIN's longitudinal mode (Thaben and Westermark, 2014).

\section{Statistical Analysis}

Statistical analyses were performed using the IBM SPSS software 20.0. The normality of data distribution was determined by the Shapiro Wilk test, and homogeneity of variances was analyzed by Levene's test. The body weight was analyzed by a two-way ANOVA test for repeated measures in one of the factors, with the Bonferroni adjustment and data were expressed as mean \pm SEM. Transcript levels between the two groups were analyzed by the Mann-Whitney $U$ test and Student's $t$-test.

\section{RESULTS}

\section{Impact of Gestational CPS on Liver Clock Genes Expression in Adult Male Offspring}

The body weight data showed that gestational CPS in both, newborn male (P1) and adult male (P90) offspring was statistically greater than in LD progeny $(\mathrm{P} 1: 7.1 \pm 0.09 \mathrm{~g}$ CPS vs. $6.78 \pm 0.07 \mathrm{~g}$ LD, unpaired $t$-test $p=0.01$; P90: $499.2 \pm 5.9$ g CPS vs. $476.2 \pm 7.9$ g LD, ANOVA two way test for repeated measures with Bonferroni adjustment $p=0.032$ ). We found no differences in the weight of female offspring at P1, size of the litters, and weight of the liver, lung, thymus, and spleen at P90 between LD and CPS (Supplementary Table 2 and Supplementary Figure 1). Next, we evaluated the effects of gestational CPS on the liver clock genes expression at the transcript level in the male offspring at P90. Our results showed daily rhythm expression of Bmall in the offspring from both conditions (Figure 2A, left and Supplementary Tables 3-5), but with significantly reduced mRNA expression in the progeny gestated under CPS conditions (Figure 2A, right). Interestingly we found that the daily peak of Clock and Nfil3 gene at transcript level was changed by $4 \mathrm{~h}$ (ZT 3.7, 4.0, and 1 to $0.4,0.0,21$ for Cosinor, JKT_Cycle and RAN, respectively) between CPS and LD gestated adult progeny (Supplementary Tables 3-5). Bmal1 and Clock are an important positive component of the central loop and promotes the expression of other clock genes. For this reason, we determined the transcript level of other genes of the central loop of the molecular clock (Clock, Per1, Per2, Per3, Cry1, and Cry2). The analysis of results showed that the daily peak of expression in the control condition (LD) of all these genes was in agreement with the reported ZT in other studies on the adult rat (Sládek et al., 2007; Figure 2B, black dots and Supplementary Tables 3-5). More importantly, the genes positively regulated by Bmal1 of the central loop evaluated here showed a similar daily rhythm of expression in both progenies with no remarkable effect on the daily rhythm pattern (Figure 2B and Supplementary Tables 3-5) or total expression (data not shown) at the mRNA level in LD and CPS male offspring.

Interlocked with the central loop there is an additional wellestablished secondary or accessory loop that involves REV-ERB and RORs transcription factors which influence negatively and positively, respectively on Bmall transcription by binding to its promoter site (RORE site), and also regulate the expression of the Nuclear Factor Interleukin 3 gene (Nfil3). Remarkable differences in the daily expression were found in two clockwork components of the accessory loop. The Ror $\alpha$ and Ror $\gamma$ components of this loop were significantly increased at the mRNA level in the CPS male offspring (Figures 3A,B). Our results showed that the transcriptional level of $\mathrm{Nfil3}$ was increased in males gestated under CPS conditions (Figure 3C), with a clear acrophase in the active phase (dark phase) of the daily expression (Figure 3C, red dots and Supplementary Tables 3-5). Interestingly, Ror $\alpha$ displayed a pattern of daily rhythm only in adult males gestated in CPS with an acrophase at active phase (Figure 3A, red dots and Supplementary Tables 3-5). Differences at individuals time points but not in the daily total expression were found in the main repressor component of the accessory loop RevErb $\alpha$ between CPS and LD male progeny (Figure 3D, right and left, respectively).

\section{Impact of Gestational CPS on the Expression of the Fibrinolytic System in the Adult Offspring}

Alterations of fibrinolytic activity mediated by deregulation in the expression of its components have been associated with a 


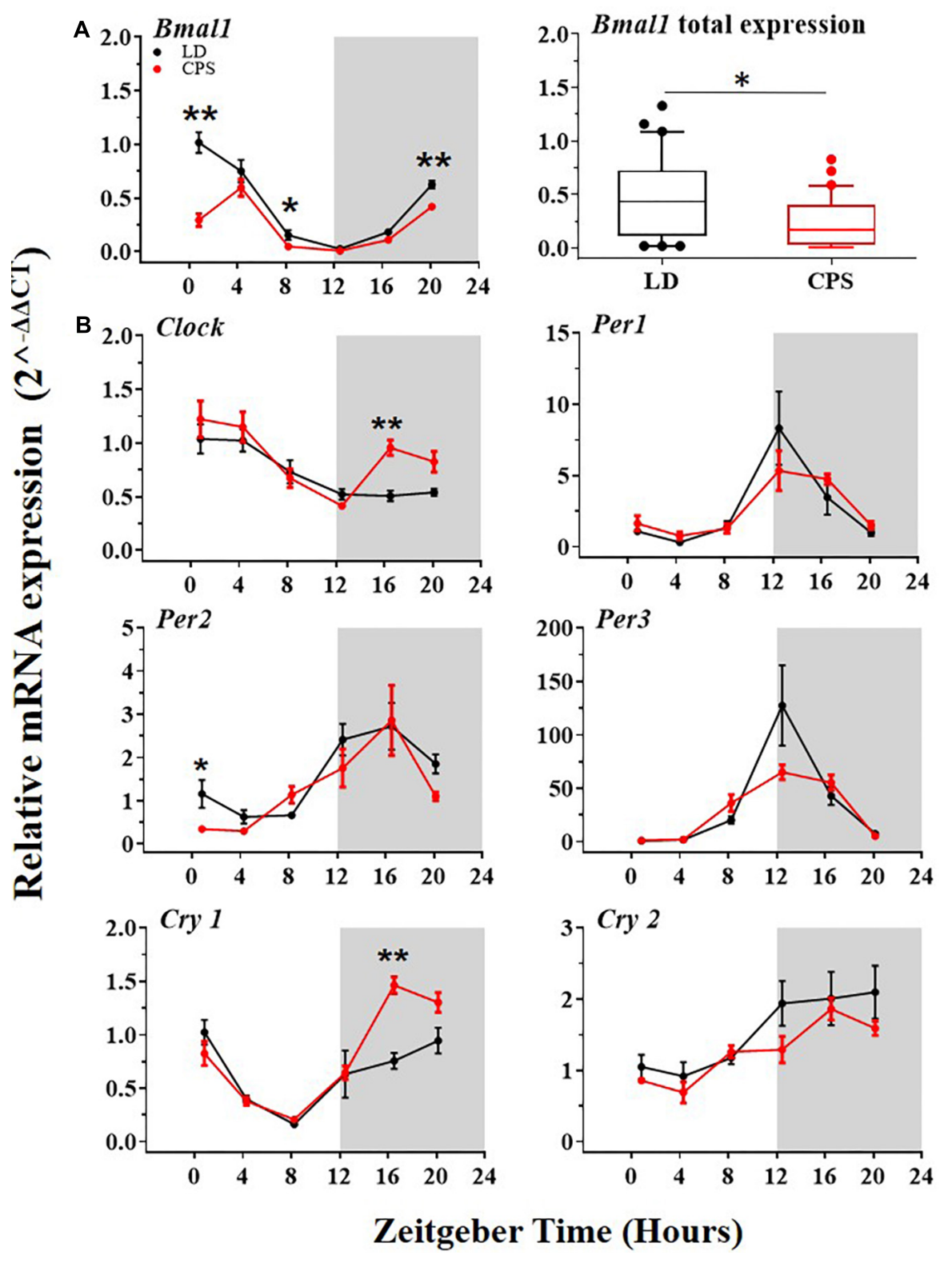

FIGURE 2 | The transcription level of clock genes of the central loop in the liver from 90-day-old male rats by RT-PCR. [A(left),B] Detection of daily rhythm. Black symbols represent males gestated under control conditions (LD, black dots), and red symbols indicate males gestated in CPS (CPS, red dots). Males from each pregnancy condition (LD: $n=12$ and CPS: $n=6$ mothers) in LD and CPS offspring ( $n=5 /$ each time point). Time is expressed as zeitgeber time (ZT), with ZTO as time lighting onset and ZT12 as lighting end; the gray bar indicates lights off. The RAIN's longitudinal mode, JTK_Cycle and the single cosinor method were used to determine daily rhythm $(p<0.05)$, solid black and red lines represent the detection of a 24-h daily rhythm for the three methods. (A,left) Data for Bmal1 are shown. ${ }^{*} p<0.05$ indicate differences between LD and CPS for time point (Mann-Whitney $U$ test). (A, right) Daily total expression. Data for Bmal1, minimum, first quartile, median, third quartile, and maximum were for LD offspring: Bmal1: 0.02, 0.11, 0.44, 0.73, and 1.33; and for CPS offspring: 0.01, 0.03, 0.17, 0.40, and 0.83. ${ }^{*} p<0.05$. Different from LD (Mann-Whitney $U$ test). (B) Data for other clock genes are shown $* p<0.05,{ }^{* *} p<0.01$ Different from LD for time point (Mann-Whitney $U$ test).

risk factor for CVD (Mavri et al., 2004; Oishi, 2009). Also, in the liver, the expression of important components of the fibrinolytic system is controlled by the circadian system. Our results showed that males gestated in CPS displayed significant differences in the mRNA expression level in important components of the fibrinolytic system relative to the LD group. More specifically, the main inhibitor of this system, Pai-1, was increased (Figure 4A, right); in contrast, the precursor of plasmin, $\mathrm{Plg}$ and $t P A$, were reduced (Figures 4B,C, right). On the other hand, $u P A$ did not show significant differences between the two progenies (Figure 4D, right). Regarding daily oscillations of mRNA components of the fibrinolytic system evaluated here, we only 


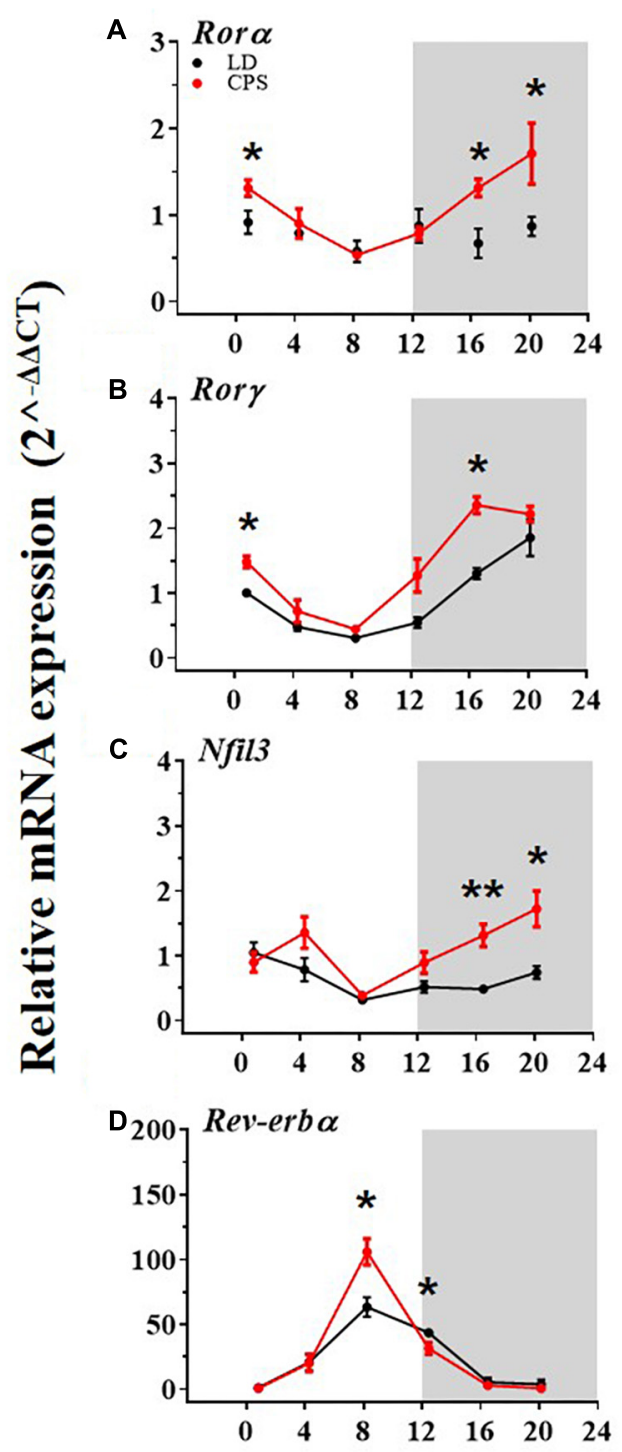

Zeitgeber Time (Hours)
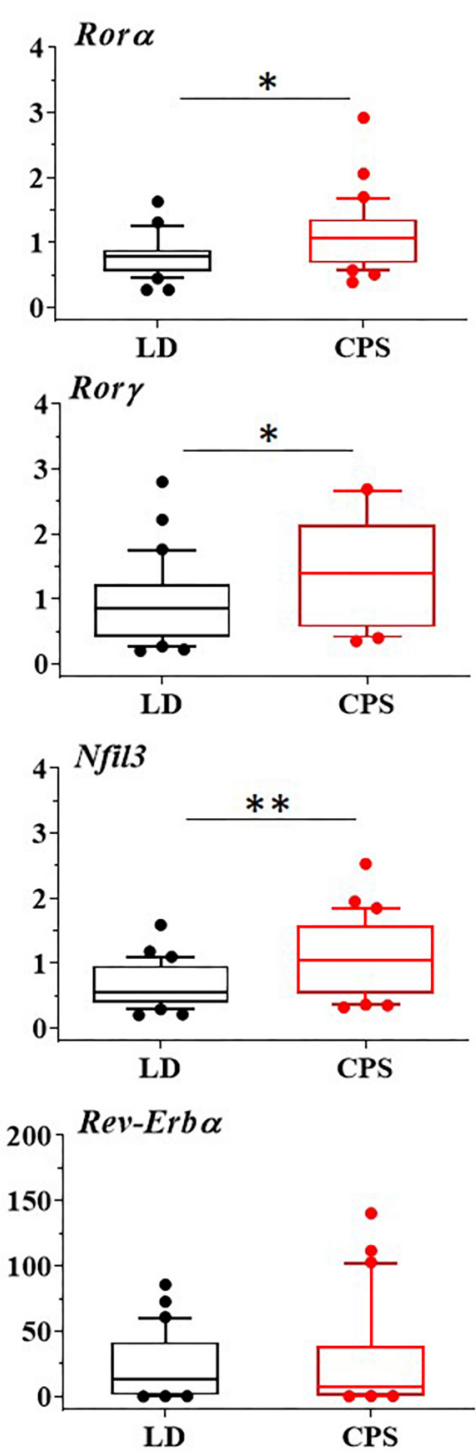

Daily total expression

FIGURE 3 | The transcription level of clock genes of the accessory loop in the liver from 90-day-old male rats by RT-PCR. (A-D,left) Detection of daily rhythm. Black symbols represent males gestated under control conditions (LD, black dots), and red symbols indicate males gestated in CPS (CPS, red dots). Males from each pregnancy condition (LD: $n=12$ and CPS: $n=6$ mothers) in LD and CPS offspring ( $n=5$ /each time point). Time is expressed as zeitgeber time (ZT), with ZTO as time lighting onset and ZT12 as lighting end; the gray bar indicates lights off. The RAIN's longitudinal mode, JTK_Cycle and the single cosinor method were used to determine daily rhythm ( $p<0.05$ ), solid black and red lines represent the detection of a $24-\mathrm{h}$ daily rhythm for the three methods. ${ }^{*} p<0.05$, ${ }^{* *} p<0.01$. Different from LD for that time point (Mann-Whitney $U$ test). (A-D,right) Daily total expression. Data and median with interquartile range are shown, in LD and CPS ( $=30$ ) offspring. ${ }^{*} p<0.05,{ }^{* *} p<0.01$. Different from LD (Mann-Whitney $U$ test). Minimum, first quartile, median, third quartile, and maximum were for LD offspring: Rora:0.27, 0.55, 0.78, 0.86, and 1.63; Rory: 0.2, 0.41, 0.86, 1.23, and 2.80; Nfil3: 0.2, 0.38, 0.55, 0.96, and 1.59; and for CPS offspring: Ror 0 : 0.39, 0.68, 1.07, 1.44, and 2.92; Ror $\gamma: 0.35,0.58,1.42,2.15$, and 5.6; Nfil3: $0.32,0.53,1.07,1.64$, and 2.53 .

detected a rhythm in tPA but only by RAIN method in CPS but not in LD adult male progeny (Figures 4A-D left, and Supplementary Tables 3-5).

At the protein level, the results obtained for daily plasma concentration of PAI-1 did not show significant differences between CPS and LD male offspring (Supplementary Figure 2 and Supplementary Table 6). However, daily rhythms were found for PAI-1 plasma protein concentration in both CPS and LD progeny; displaying a daily peak expression of the protein in the active phase (dark) of the circadian cycle (Ohkura et al., 2006). Notably, at P90 of development the amplitude of the oscillation of PAI-1 plasma concentration was increased in 


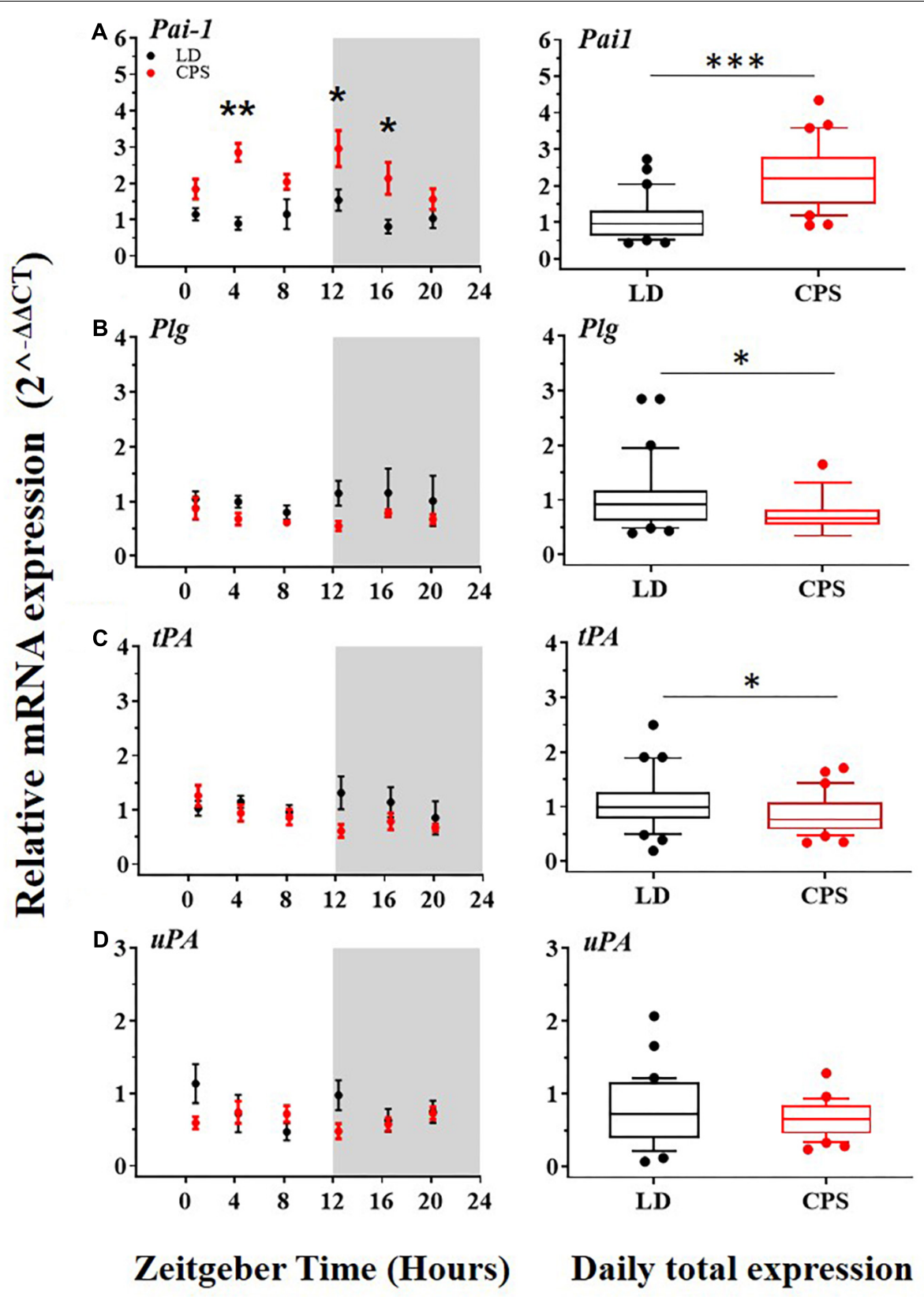

FIGURE 4 | The transcription level of Pai-1, Plg, tPA, and uPA in the liver from 90-day-old male rats by RT-PCR. (A-D,left) Detection of daily rhythm. Black symbols represent males gestated under control conditions (LD, black dots) and red symbols indicate males gestated in CPS (CPS, red dots). Males from each pregnancy condition (LD: $n=12$ and CPS: $n=6$ mothers), in LD and CPS offspring ( $n=5$ /each time point). Time is expressed as zeitgeber time (ZT), with ZT0 as time lighting onset and ZT12 as lighting end; the gray bar indicates lights off. The RAIN's longitudinal mode, JTK_Cycle and the single cosinor method were used to determine daily rhythm $(p<0.05)$, solid black and red lines represent the detection of a 24-h daily rhythm for the three methods. (A-D,right) Daily total expression. Data and median with interquartile range are shown in LD and CPS $(n=30)$ offspring. ${ }^{*} p<0.05,{ }^{* *} p<0.01,{ }^{* * *} p<0.001$. Different from LD (Mann-Whitney $U$ test). Minimum, first quartile, median, third quartile, and maximum for LD offspring: Pai-1: 0.44, 0.62, 0.96, 1.33, and 2.73; Plg: 0.39, 0.61, 0.92, 1.15, and 2.85; tPA: 0.19, $0.78,0.99,1.27$, and 2.5 and for CPS offspring: Pai-1: 0.92, 1.5, 2.27, 3.01, and 7.76; Plg: 0.34, 0.55, 0.66, 0.81, and 1.65; tPA: 0.34, 0.58, 0.76, 1.09, and 1.7.

adult males which had been gestated under CPS $(\mathrm{A}=198.8$ (LD) and 387.9 (CPS); AMP = 96.0 (LD) and 206.0 (CPS) determined by Cosinor and JTK_Cycle, respectively) relative to LD adult offspring (Supplementary Table 6). Finally, we found a circadian rhythm in the ex vivo coagulation time in both CPS and LD progeny (Supplementary Figure 3 and Supplementary Tables 7-9). Remarkably, at the postnatal age of 180 days, in CPS gestated male the time required to ex vivo coagulation was greater than LD. This difference showed seems to be age-dependent because it was not observed at P60 or P120 
(Supplementary Figures 3A,B). These results point to a putative mayor propensity to a coagulation/fibrinolytic system imbalance in CPS than LD during the aging process.

\section{DISCUSSION}

Modern lifestyles are strongly correlated with misalignment of biological clocks. In this context, circadian disruption act as a sustained environmental factor that leads to conflicts between endogenous biologic clock cycles and the environment (Bass and Lazar, 2016). In the present study, gene expression patterns in the peripheral liver clock and fibrinolytic system were assessed to determine the long-term effects of gestational chronic photoperiod shifting (mimicking repeated night shift work schedules in pregnant women) on the adult offspring.

Hormonal disturbances have also been linked to altered photoperiods. Impaired secretion of corticosterone, aldosterone, and the loss of response to ACTH of the adrenal gland have been observed in progeny gestated under CPS (Mendez et al., 2016; Salazar et al., 2018). Adrenal function is directly regulated by the photoperiod as it is strictly controlled by the master clock residing in the suprachiasmatic nucleus (Aoshima et al., 2014; Plano et al., 2017). Moreover, the adrenal gland is an important oscillator from fetal to postnatal period of life (Torres-Farfan et al., 2011; Roa et al., 2017; Salazar et al., 2018) that synchronizes the rhythmic signaling of glucocorticoids and catecholamines to peripheral clocks such as the liver (Kalsbeek et al., 2012; Pezük et al., 2012). In fact, a significant desynchronization is observed in the liver of adult rats subjected to adrenalectomy (Pezük et al., 2012), strongly suggesting that the adrenal peripheral oscillator plays a crucial role in synchronizing the circadian rhythm of the liver. Previous findings in adult rats gestated under CPS indicate significant desynchronization of daily rhythms of plasma corticosterone, whereas the daily pattern of plasma ACTH was similar in both CPS and control offspring; however, corticosterone response to ACTH was lost in CPS adrenals (Mendez et al., 2016; Salazar et al., 2018). These lines of evidence could be associated with the alteration of the hepatic circadian clock. In the liver, our results demonstrated that transcript levels of Bmall and the phase of the daily peak expression of Clock and Nfil3 were significantly affected in adult males gestated in CPS. Interestingly, gestational CPS disrupted daily rhythms in the liver of these clock-genes even after 3 months of exposure to LD photoperiod during the postnatal developing (P90). These results reveal a long-term effect on the expression of the clock genes that changes the phenotype displayed at the adult stage under LD photoperiod in a male gestated in CPS protocol. Importantly, Bmall plays a key role in the regulation of the hemostatic function of the liver and also in the progression of the prothrombotic state in aging (Hemmeryckx et al., 2011, 2019).

Alterations of the molecular clock at the accessory loop (RORE site) in the male adult progeny were also evidenced. Specifically, transcript levels of the clock genes Ror $\alpha$ and Ror $(\operatorname{Ror} \alpha / \gamma)$ were increased in adult CPS males. The expression of Ror $\alpha / \gamma$ clock genes has been described to be positively controlled by the BMAL/CLOCK heterodimer. However, as it was described before, the transcript levels of Bmal1 were downregulated in adult males gestated under CPS relative to LD conditions. That can be explained by the fact that posttranslational modifications of the BMAL/CLOCK heterodimer have been shown play a key role in terms to modify its activity independently of mRNA level regulation (Hirayama et al., 2007; Bellet and SassoneCorsi, 2010; Preußner and Heyd, 2016). Additionally, it has been demonstrated that Ror $\alpha / \gamma$ expression is controlled by other circadian signals via cAMP response elements (CREs) (O'Neill et al., 2008) that could increase its role under the effects of gestational CPS. In order to determine if increased transcript levels of Ror $\alpha / \gamma$ may have functional consequences, we evaluated transcript levels of Nfil3 in the liver, because its expression is principally regulated by Ror $\gamma$ (Ueda et al., 2005; Takeda et al., 2012). The transcription level of Nfil3 was significantly increased in adult males gestated in CPS. Also, the phase (ZT) of the daily peak expression of $N f i l 3$ was significantly affected $(3.5 \mathrm{~h})$ in males gestated in CPS (Supplementary Tables 3-5). This finding emphasizes that the effects on the accessory loop of the molecular clock could deregulate CCGs and therefore alter physiological functions. Moreover, endocrine signal as insulin is also important in the regulation of the expression of Nfil3 (Keniry et al., 2014) and as previously mentioned, hyperinsulinemia has been reported in adult offspring gestated in CPS (Varcoe et al., 2011).

A reduced fibrinolytic activity due to an increase in the expression of PAI-1 is a characteristic risk factor for CVD (Mavri et al., 2004; Oishi, 2009). Liver physiology is heavily involved in the regulation of fibrinolytic activity since many of its components, like plasminogen (Cesarman-Maus and Hajjar, 2005; Leebeek and Rijken, 2015) and PAI-1 (Oishi, 2009; Declerck and Gils, 2013) are mainly synthesized by this organ. In adult mice, it has been reported that chronic alteration of the photoperiod was associated with the deregulation of the Pai-1 expression in the liver (Oishi and Ohkura, 2013). On the other hand, previous evidence indicates that clock genes regulate the expression of PAI-1 (Schoenhard et al., 2003; Ohkura et al., 2006; Wang et al., 2006). For instance, a mouse model deficient in Bmal1 (Bmal1 ${ }^{-/-}$) displayed elevated plasma levels of PAI-1, which were associated with a prothrombotic phenotype (Hemmeryckx et al., 2011; Somanath et al., 2011). Regarding gestational chronodisruption, our results showed increased levels of Pai-1 in the liver of male adult offspring gestated under CPS. This observation is relevant because we previously showed a significant increase in blood pressure in CPS males at P90 (Mendez et al., 2016). Both, increased levels of PAI-1 associated with clock genes deregulation here reported and high pressure described previously are recognized like a CVD risk factors. Some factors that induce Pai-1 gene expression are insulin, glucocorticoids (Irigoyen et al., 1999; Mavri et al., 2004; Dimova and Kietzmann, 2008). Interestingly, factors as hyperinsulinemia and alteration of corticosterone circadian rhythm also described in this animal model (Varcoe et al., 2011; Mendez et al., 2016) have been shown to induce greater expression of Pai-1 in the liver. On the other hand, our data showed a decreased expression of Bmall clock gene in the males gestated in CPS. Previous evidence supports the idea that reduced expression of Bmall in the liver results in increased expression levels of Pai-1. In particular, 
the upregulation of PAI-1 is associated with an increase in thrombosis propensity during the aging process (Hemmeryckx et al., 2011, 2019; Somanath et al., 2011). In addition, REV-ERB $\alpha$ is a negative regulator of Pai-1 (Wang et al., 2006) expression by a mechanism involving its competition with $\mathrm{ROR} \alpha$, a positive regulator that our results showed that is increased at transcript level and also rhythmic with a phase (ZT) of the daily peak expression in the active phase (dark) in CPS adult progeny. We did not observe differences in Rev-Erb $\alpha$ daily total expression between adult males gestated under CPS and LD conditions, suggesting an inclination to induce Pai- 1 expression by $\mathrm{ROR} \alpha$ rather than repression by REV-ERB $\alpha$.

The appearance of a marked daily oscillation at the transcript level of the clock gene Ror $\alpha$ in adult CPS but not in LD offspring was another interesting finding. The induction of rhythmic expression of genes that are not oscillatory could be mediated by epigenetic mechanisms, which are involved in the regulation of the transcriptional machinery and reveal that expression of genes that are not rhythmic could be induced (Masri et al., 2014). The induction of the rhythmic expression pattern in Ror $\alpha$ in CPS adult males suggests that epigenetic mechanisms might play a role in the long-term effects observed.

At the protein level, results obtained for daily plasma concentration of PAI-1 did not show significant differences between CPS and LD male offspring. However, daily rhythms were found for PAI-1 plasma protein; displaying a daily peak expression of the protein in the active phase (dark) of the daily cycle that are in agreement with data previously reported in rodents for LD condition (Ohkura et al., 2006). Notably, at P90 of development, the amplitude of the oscillation of plasma concentration in the active phase was increased in adult males which had been gestated under CPS relative to LD offspring (Supplementary Table 6).

It has been described that older humans are more susceptible to thrombosis under septic conditions (Balleisen et al., 1985; Aillaud et al., 1986). In addition, murine models have been demonstrated that the aging process increases the endotoxininduced thrombosis by a mechanism that involves increased expression of PAI-1 protein in the plasma and at mRNA level in the liver. This tendency is linked to an enhanced inflammatory response in aged mice (Yamamoto et al., 2002). Connected with this previous literature we found a circadian rhythm in the ex vivo

\section{REFERENCES}

Aillaud, M. F., Pignol, F., Alessi, M. C., Harle, J. R., Escande, M., Mongin, M., et al. (1986). Increase in plasma concentration of plasminogen activator inhibitor, fibrinogen, von Willebrand factor, factor VIII:C and in erythrocyte sedimentation rate with age. Thromb. Haemost. 55, 330-332. doi: 10.1055/s0038- 1661557

Albrecht, U. (2012). Timing to perfection: the biology of central and peripheral circadian clocks. Neuron 74, 246-260. doi: 10.1016/j.neuron.2012. 04.006

Amaral, F. G., Castrucci, A. M., Cipolla-Neto, J., Poletini, M. O., Mendez, N., Richter, H. G., et al. (2014). Environmental control of biological rhythms: effects on development, fertility and metabolism. J. Neuroendocrinol. 26, 603-612. doi: 10.1111/jne. 12144 coagulation time that was increased at postnatal age of 180 days in CPS gestated male in a process that is age-dependent because this difference was not observed at P60 or P120.

\section{DATA AVAILABILITY STATEMENT}

The datasets generated for this study are available on request to the corresponding author.

\section{ETHICS STATEMENT}

The animal study was reviewed and approved by Bioethics Commission of the Universidad Austral de Chile (CBA number 267/2016).

\section{AUTHOR CONTRIBUTIONS}

PC and JS contributed to the conception, design, and drafting of the work. PC wrote the first draft of the manuscript. PC, BP, CT, $\mathrm{NM}, \mathrm{GE}$, and $\mathrm{KV}$ worked on animal handling and acquisition of data. PC, JS, and FM analyzed and interpreted the data. CT-F, $\mathrm{HR}$, and $\mathrm{PB}$ critically reviewed the manuscript for important intellectual content. All authors contributed to the manuscript revision, and read and approved the submitted version.

\section{FUNDING}

This work was supported by Grant $\mathrm{N}^{\circ} 1150789$ from the Fondo Nacional de Desarrollo Científico y Tecnológico (FONDECYT), Chile, and ANILLO ACT-1116. PC was supported by Fellowship $N^{\circ} 21171387$ from the Comisión Nacional de Investigación Científica y Tecnológica (CONICYT), Chile.

\section{SUPPLEMENTARY MATERIAL}

The Supplementary Material for this article can be found online at: https://www.frontiersin.org/articles/10.3389/fphys. 2019.01377/full\#supplementary-material

Aoshima, Y., Sakakibara, H., Suzuki, T., Yamazaki, S., and Shimoi, K. (2014). Nocturnal light exposure alters hepatic Pai-1 expression by stimulating the adrenal pathway in C3H mice. Exp. Anim. 63, 331-338. doi: 10.1538/expanim. 63.331

Balleisen, L., Assmann, G., Bailey, J., Epping, P.-H., Schulte, H., and van de Loo, J. (1985). Epidemiological study on factor VII, factor VIII and fibrinogen in an industrial population-II. Baseline data on the relation to blood pressure, blood glucose, uric acid, and lipid fractions. Thromb. Haemost. 54, 721-723. doi: $10.1055 / \mathrm{s}-0038-1660106$

Bass, J., and Lazar, M. A. (2016). Transformed more than 14 years later with posi- tional cloning of core clock genes and recognition. Science 80:354. doi: 10.1126/science.aah4965

Bellet, M. M., and Sassone-Corsi, P. (2010). Mammalian circadian clock and metabolism - the epigenetic link. J. Cell Sci. 123, 3837-3848. doi: 10.1242/jcs. 051649 
Calkins, K., Devaskar, S. U., and Angeles, L. (2011). Fetal origins of adult disease. Curr. Probl. Pediatr. Adolesc. Health Care 41, 158-176. doi: 10.1016/j.cppeds. 2011.01.001.Fetal

Capra, L., Tezza, G., Mazzei, F., and Boner, A. L. (2013). The origins of health and disease: the influence of maternal diseases and lifestyle during gestation. Ital. J. Pediatr. 39:7. doi: 10.1186/1824-7288-39-7

Čečmanová, V., Houdek, P., Šuchmanová, K., Sládek, M., and Sumová, A. (2019). Development and entrainment of the fetal clock in the suprachiasmatic nuclei: the role of glucocorticoids. J. Biol. Rhythms 34, 307-322. doi: 10.1177/ 0748730419835360

Cesarman-Maus, G., and Hajjar, K. A. (2005). Molecular mechanisms of fibrinolysis. Br. J. Haematol. 129, 307-321. doi: 10.1111/j.1365-2141.2005. 05444.x

Curtis, A. M., Bellet, M. M., Sassone-Corsi, P., and O’Neill, L. A. J. (2014). Circadian clock proteins and immunity. Immunity 40, 178-186. doi: 10.1016/ j.immuni.2014.02.002

Declerck, P. J., and Gils, A. (2013). Three decades of research on plasminogen activator inhibitor-1: a multifaceted serpin. Semin. Thromb. Hemost. 39, 356364. doi: 10.1055/s-0033-1334487

Dimova, E. Y., and Kietzmann, T. (2008). Metabolic, hormonal and environmental regulation of plasminogen activator inhibitor-1 (PAI-1) expression: lessons from the liver. Thromb. Haemost. 100, 992-1006. doi: 10.1160/TH08-07-0490

Oishi, K., and Ohkura, N. (2013). Chronic circadian clock disruption induces expression of the cardiovascular risk factor plasminogen activator inhibitor1 in mice. Blood Coagul. Fibrinolysis 24, 106-108. doi: 10.1097/MBC. 0b013e32835bfdf3

Erren, T. C., and Reiter, R. J. (2009). Defining chronodisruption. J. Pineal Res. 46, 245-247. doi: 10.1111/j.1600-079X.2009.00665.x

Fowden, A. L., Giussani, D. A., and Forhead, A. J. (2006). Intrauterine programming of physiological systems: causes and consequences. Physiology 21, 29-37. doi: 10.1152/physiol.00050.2005

Gatford, K. L., Kennaway, D. J., Liu, H., Kleemann, D. O., Kuchel, T. R., and Varcoe, T. J. (2019). Simulated shift work disrupts maternal circadian rhythms and metabolism, and increases gestation length in sheep. J. Physiol. 579, 1889-1904. doi: $10.1113 /$ JP277186

Hanson, M. A., and Gluckman, P. D. (2014). Early developmental conditioning of later health and disease: physiology or pathophysiology? Physiol. Rev. 94, 1027-1076. doi: 10.1152/physrev.00029.2013

Haus, E. (2007). Chronobiology of hemostasis and inferences for the chronotherapy of coagulation disorders and thrombosis prevention. $A d v$. Drug Deliv. Rev. 59, 966-984. doi: 10.1016/j.addr.2006.11.002

Hemmeryckx, B., Frederix, L., and Lijnen, H. R. (2019). Deficiency of bmall disrupts the diurnal rhythm of haemostasis. Exp. Gerontol. 118, 1-8. doi: 10. 1016/J.EXGER.2018.12.017

Hemmeryckx, B., Van Hove, C. E., Fransen, P., Emmerechts, J., Kauskot, A., Bult, H., et al. (2011). Progression of the prothrombotic state in aging bmall-deficient mice. Arterioscler. Thromb. Vasc. Biol. 31, 2552-2559. doi: 10.1161/ATVBAHA. 111.229062

Hirayama, J., Sahar, S., Grimaldi, B., Tamaru, T., Takamatsu, K., Nakahata, Y., et al. (2007). CLOCK-mediated acetylation of BMAL1 controls circadian function. Nature 450, 1086-1090. doi: 10.1038/nature06394

Hughes, M. E., Abruzzi, K. C., Allada, R., Anafi, R., Arpat, A. B., Asher, G., et al. (2017). Guidelines for genome-scale analysis of biological rhythms. J. Biol. Rhythms 32, 380-393. doi: 10.1177/0748730417728663

Hughes, M. E., Hogenesch, J. B., and Kornacker, K. (2010). JTK_CYCLE: an efficient non-parametric algorithm for detecting rhythmic components in genome-scale datasets. J. Biol. Rhythms 25:372. doi: 10.1177/0748730410379711

Irigoyen, J. P., Munoz-Canoves, P., Montero, L., Koziczak, M., and Nagamine, Y. (1999). The plasminogen activator system:biology and regulation. Cell. Mol. Life Sci. 56, 104-132.

Jung, R. G., Simard, T., Labinaz, A., Ramirez, F. D., Di Santo, P., Motazedian, P., et al. (2018). Role of plasminogen activator inhibitor-1 in coronary pathophysiology. Thromb. Res. 164, 54-62. doi: 10.1016/j.thromres.2018. 02.135

Kalsbeek, A., van der Spek, R., Lei, J., Endert, E., Buijs, R. M., and Fliers, E. (2012). Circadian rhythms in the hypothalamo-pituitary-adrenal (HPA) axis. Mol. Cell. Endocrinol. 349, 20-29. doi: 10.1016/J.MCE.2011.06.042
Keniry, M., Dearth, R. K., Persans, M., and Parsons, R. (2014). New frontiers for the NFIL3 bZIP transcription factor in cancer. Metabol. Beyond. Discover. 2:e15. doi: $10.15190 /$ d.2014.7

Leebeek, F. W. G., and Rijken, D. C. (2015). The fibrinolytic status in liver diseases. Semin. Thromb. Hemost. 41, 474-480. doi: 10.1055/s-0035-1550437

Liu, Z., and Chu, G. (2013). Chronobiology in mammalian health. Mol. Biol. Rep. 40, 2491-2501. doi: 10.1007/s11033-012-2330-4

Livak, K. J., and Schmittgen, T. D. (2001). Analysis of relative gene expression data using real-time quantitative PCR and. Methods 25, 402-408. doi: 10.1006/meth. 2001.1262

Maemura, K., de la Monte, S. M., Chin, M. T., Layne, M. D., Hsieh, C. M., Yet, S. F., et al. (2000). CLIF, a novel cycle-like factor, regulates the circadian oscillation of plasminogen activator inhibitor-1 gene expression. J. Biol. Chem. 275, 36847-36851. doi: 10.1074/jbc.C000629200

Marsh, L. M., Pfefferle, P. I., Pinkenburg, O., and Renz, H. (2011). Maternal signals for progeny prevention against allergy and asthma. Cell. Mol. Life Sci. 68, 1851-1862. doi: 10.1007/s00018-011-0644-3

Masri, S., Rigor, P., Cervantes, M., Ceglia, N., Sebastian, C., Xiao, C., et al. (2014). Partitioning circadian transcription by SIRT6 leads to segregated control of cellular metabolism. Cell 158, 659-672. doi: 10.1016/j.cell.2014.06.050

Mavri, A., Alessi, M. C., and Juhan-Vague, I. (2004). Hypofibrinolysis in the insulin resistance syndrome: implication in cardiovascular diseases. J. Intern. Med. 255, 448-456. doi: 10.1046/j.1365-2796.2003.01288.x

Mazzoccoli, G., Pazienza, V., and Vinciguerra, M. (2012). Clock genes and clockcontrolled genes in the regulation of metabolic rhythms. Chronobiol. Int. 29, 227-251. doi: 10.3109/07420528.2012.658127

Mendez, N., Halabi, D., Spichiger, C., Salazar, E. R., Vergara, K., Alonso-Vasquez, P., et al. (2016). Gestational chronodisruption impairs circadian physiology in rat male offspring, increasing the risk of chronic disease. Endocrinology 157, 4654-4668. doi: 10.1210/en.2016-1282

Mohawk, J. A., Green, C. B., and Takahashi, J. S. (2013). Central and peripheral circadian clocks in mammal. Annu. Rev. Neurosci. 35, 445-462. doi: 10.1146/ annurev-neuro-060909-153128.CENTRAL

Ohkura, N., Oishi, K., Fukushima, N., Kasamatsu, M., Atsumi, G. I., Ishida, N., et al. (2006). Circadian clock molecules CLOCK and CRYs modulate fibrinolytic activity by regulating the PAI-1 gene expression. J. Thromb. Haemost. 4, $2478-$ 2485. doi: 10.1111/j.1538-7836.2006.02210.x

Oishi, K. (2009). Plasminogen activator inhibitor-1 and the circadian clock in metabolic disorders. Clin. Exp. Hypertens. 31, 208-219. doi: 10.1080/ 10641960902822468

O’Neill, J. S., Maywood, E. S., Chesham, J. E., Takahashi, J. S., and Hastings, M. H. (2008). cAMP-dependent signaling as a core component of the mammalian circadian pacemaker. Science 320, 949-953. doi: 10.1126/science.1152506

Partch, C. L., Green, C. B., and Takahashi, J. S. (2014). Molecular architecture of the mammalian circadian clock. Trends Cell Biol. 24, 90-99. doi: 10.1016/j.tcb. 2013.07.002

Pezük, P., Mohawk, J. A., Wang, L. A., and Menaker, M. (2012). Glucocorticoids as entraining signals for peripheral circadian oscillators. Endocrinology 153, 4775-4783. doi: 10.1210/en.2012-1486

Plano, S. A., Casiraghi, L. P., Moro, P. G., Paladino, N., Golombek, D. A., and Chiesa, J. J. (2017). Circadian and metabolic effects of light: implications in weight homeostasis and health. Front. Neurol. 8:1-21. doi: 10.3389/fneur.2017. 00558

Preußner, M., and Heyd, F. (2016). Post-transcriptional control of the mammalian circadian clock: implications for health and disease. Pflugers Arch. Eur. J. Physiol. 468, 983-991. doi: 10.1007/s00424-016-1820-y

Refinetti, R., Cornélissen, G., and Halberg, F. (2007). Procedures for numerical analysis of circadian rhythms. Biol. Rhythm Res. 38, 275-325. doi: 10.1080/ 09291010600903692

Reinke, H., and Asher, G. (2016). Circadian clock control of liver metabolic functions. Gastroenterology 150, 574-580. doi: 10.1053/j.gastro.2015.11.043

Richter, H. G., Mendez, N., Abarzua-Catalan, L., Valenzuela, G. J., Seron-Ferre, M., and Torres-Farfan, C. (2018). Developmental programming of capuchin monkey adrenal dysfunction by gestational chronodisruption. Biomed. Res. Int. 2018:11. doi: 10.1155/2018/9183053

Roa, S. L. R., Martinez, E. Z., Martins, C. S., Antonini, S. R., de Castro, M., and Moreira, A. C. (2017). Postnatal ontogeny of the circadian expression of the 
adrenal clock genes and corticosterone rhythm in male rats. Endocrinology 158, 1339-1346. doi: 10.1210/en.2016-1782

Salazar, E. R., Richter, H. G., Spichiger, C., Mendez, N., Halabi, D., Vergara, K., et al. (2018). Gestational chronodisruption leads to persistent changes in the rat fetal and adult adrenal clock and function. J. Physiol. 596, 5839-5857. doi: 10.1113/JP276083

Schoenhard, J. A., Smith, L. H., Painter, C. A., Eren, M., Johnson, C. H., and Vaughan, D. E. (2003). Regulation of the PAI-1 promoter by circadian clock components: differential activation by BMAL1 and BMAL2. J. Mol. Cell. Cardiol. 35, 473-481. doi: 10.1016/S0022-2828(03)00051-8

Serón-Ferré, M., Forcelledo, M. L., Torres-Farfan, C., Valenzuela, F. J., Rojas, A., Vergara, M., et al. (2013). Impact of chronodisruption during primate pregnancy on the maternal and newborn temperature rhythms. PLoS One 8:e57710. doi: 10.1371/journal.pone.0057710

Serón-Ferré, M., Mendez, N., Abarzua-Catalan, L., Vilches, N., Valenzuela, F. J., Reynolds, H. E., et al. (2012). Circadian rhythms in the fetus. Mol. Cell. Endocrinol. 349, 68-75. doi: 10.1016/J.MCE.2011.07.039

Sládek, M., Jindráková, Z., Bendová, Z., and Sumová, A. (2007). Postnatal ontogenesis of the circadian clock within the rat liver. Am. J. Physiol. Regul. Integr. Comp. Physiol. 292, R1224-R1229. doi: 10.1152/ajpregu.00184.2006

Somanath, P. R., Podrez, E. A., Chen, J., Ma, Y., Marchant, K., Antoch, M., et al. (2011). Deficiency in core circadian protein bmall is associated with a prothrombotic and vascular phenotype. J. Cell. Physiol. 226, 132-140. doi: $10.1002 /$ jcp. 22314

Sumová, A., Bendová, Z., Sládek, M., El-Hennamy, R., Matejů, K., Polidarová, L., et al. (2008). Circadian molecular clocks tick along ontogenesis. Physiol. Res. 57, S139-S148.

Tahara, Y., and Shibata, S. (2016). Circadian rhythms of liver physiology and disease: experimental and clinical evidence. Nat. Rev. Gastroenterol. Hepatol. 13, 217-226. doi: 10.1038/nrgastro. 2016.8

Takahashi, J. S. (2016). "Molecular architecture of the circadian clock in mammals," in A Time for Metabolism and Hormones, eds P. Sassone and Y. Corsi Christen (Berlin: Springer), 13-24. doi: 10.1007/978-3-319-27069-2_2

Takeda, Y., Jothi, R., Birault, V., and Jetten, A. M. (2012). RORc directly regulates the circadian expression of clock genes and downstream targets in vivo. Nucleic Acids Res. 40, 8519-8535. doi: 10.1093/nar/gks630

Tarquini, R., and Mazzoccoli, G. (2017). Clock genes, metabolism, and cardiovascular risk. Heart Fail. Clin. 13, 645-655. doi: 10.1016/J.HFC.2017.05.001

Thaben, P. F., and Westermark, P. O. (2014). Detecting rhythms in time series with RAIN. J. Biol. Rhythms 29, 391-400. doi: 10.1177/0748730414553029

Thompson, M. D., and Trask, A. J. (2016). Developmental origins of cardiovascular dysfunction: doomed from birth? Circ J. 80, 818-820. doi: 10.1253/circj.CJ-160207

Tofler, G. H., Massaro, J., O’Donnell, C. J., Wilson, P. W. F., Vasan, R. S., Sutherland, P. A., et al. (2016). Plasminogen activator inhibitor and the risk of cardiovascular disease: the framingham heart study. Thromb. Res. 140, 30-35. doi: 10.1016/j.thromres.2016.02.002

Torres-Farfan, C., Mendez, N., Abarzua-Catalan, L., Vilches, N., Valenzuela, G. J., and Seron-Ferre, M. (2011). A circadian clock entrained by melatonin is ticking in the rat fetal adrenal. Endocrinology 152, 1891-1900. doi: 10.1210/en.20101260

Touitou, Y., Reinberg, A., and Touitou, D. (2017). Association between light at night, melatonin secretion, sleep deprivation, and the internal clock: health impacts and mechanisms of circadian disruption. Life Sci. 173, 94-106. doi: 10.1016/j.lfs.2017.02.008

Ueda, H. R., Hayashi, S., Chen, W., Sano, M., Machida, M., Shigeyoshi, Y., et al. (2005). System-level identification of transcriptional circuits underlying mammalian circadian clocks. Nat. Genet. 37, 187-192. doi: 10.1038/ng1504

Varcoe, T. J., Boden, M. J., Voultsios, A., Salkeld, M. D., Rattanatray, L., and Kennaway, D. J. (2013). Characterisation of the maternal response to chronic phase shifts during gestation in the rat: implications for fetal metabolic programming. PLoS One 8:e53800. doi: 10.1371/journal.pone.0053800

Varcoe, T. J., Gatford, K. L., and Kennaway, D. J. (2017). Maternal circadian rhythms and the programming of adult health and disease. Am. J. Physiol. Regul. Integr. Comp. Physiol 314, R231-R241. doi: 10.1152/ajpregu.00248.2017

Varcoe, T. J., Wight, N., Voultsios, A., Salkeld, M. D., and Kennaway, D. J. (2011). Chronic phase shifts of the photoperiod throughout pregnancy programs glucose intolerance and insulin resistance in the rat. PLoS One 6, 1-10. doi: 10.1371/journal.pone.0018504

Wang, J., Yin, L., and Lazar, M. A. (2006). The orphan nuclear receptor Rev-erb $\alpha$ regulates circadian expression of plasminogen activator inhibitor type. J. Biol. Chem. 281, 33842-33848. doi: 10.1074/jbc.M607873200

Yamamoto, K., Shimokawa, T., Yi, H., Isobe, K.-i., Kojima, T., Loskutoff, D. J., et al. (2002). Aging accelerates endotoxin-induced thrombosis: increased responses of plasminogen activator inhibitor-1 and lipopolysaccharide signaling with aging. Am. J. Pathol. 161, 1805-1814. doi: 10.1016/s0002-9440(10)64457-4

Zwighaft, Z., Reinke, H., and Asher, G. (2016). The liver in the eyes of a chronobiologist. J. Biol. Rhythms 31, 115-124. doi: 10.1177/0748730416633552

Conflict of Interest: The authors declare that the research was conducted in the absence of any commercial or financial relationships that could be construed as a potential conflict of interest.

Copyright (c) 2019 Carmona, Pérez, Trujillo, Espinosa, Miranda, Mendez, TorresFarfan, Richter, Vergara, Brebi and Sarmiento. This is an open-access article distributed under the terms of the Creative Commons Attribution License (CC BY). The use, distribution or reproduction in other forums is permitted, provided the original author(s) and the copyright owner(s) are credited and that the original publication in this journal is cited, in accordance with accepted academic practice. No use, distribution or reproduction is permitted which does not comply with these terms. 